\title{
A NOTE ON THE JACOBI THETA FORMULA
}

\author{
BY L. CARLITZ ${ }^{1}$
}

Communicated by Walter Rudin, June 8, 1962

In this note we show that Jacobi's identity [1, p. 280]

$$
\prod_{n=1}^{\infty}\left(1-q^{2 n}\right)\left(1+q^{2 n-1} t\right)\left(1+q^{2 n-1} t^{-1}\right)=\sum_{n=-\infty}^{\infty} q^{n^{2}} t^{n}
$$

implies relations between various partition functions of two arguments, namely (5), (7) and (8) below.

In (1) take

$$
q^{2}=x y, \quad t=x y^{-1}, \quad|x y|<1 .
$$

Then (1) becomes

(2) $\prod_{n=1}^{\infty}\left(1-x^{n} y^{n}\right)\left(1+x^{n} y^{n-1}\right)\left(1+x^{n-1} y^{n}\right)=\sum_{n=-\infty}^{\infty} x^{n(n+1) / 2} y^{n(n-1) / 2}$.

Let $\alpha(n, m)$ denote the number of partitions of $(n, m)$ into distinct parts

$$
(a, a-1), \quad(b-1, b) \quad(a, b=1,2,3, \cdots),
$$

so that we have the generating function

$$
\sum_{n, m=0}^{\infty} \alpha(n, m) x^{n} y^{m}=\prod_{n=1}^{\infty}\left(1+x^{n} y^{n-1}\right)\left(1+x^{n-1} y^{n}\right) .
$$

Then by (2) and (3)

$$
\sum_{n, m=0}^{\infty} \alpha(n, m) x^{n} y^{m}=\prod_{n=1}^{\infty}\left(1-x^{n} y^{n}\right)^{-1} \sum_{r=-\infty}^{\infty} x^{r(r+1) / 2} y^{r(r-1) / 2} .
$$

Since

$$
\prod_{n=1}^{\infty}\left(1-x^{n} y^{n}\right)^{-1}=\sum_{n=0}^{\infty} p(n) x^{n} y^{n}
$$

where $p(n)$ is the number of unrestricted partitions of $n$, it follows from (4) that

$$
\alpha(n, m)=p\left(n-\frac{1}{2}(n-m)(n-m+1)\right) .
$$

\footnotetext{
${ }^{1}$ Supported in part by National Science Foundation grant G 16485.
} 
It is understood in this formula that $p(-m)=0$ for $m>0$.

Thus the relation (5) is equivalent to Jacobi's identity (1).

In (2) replace $x, y$ by $-x,-y$, respectively, so that

(6)

$$
\begin{aligned}
\prod_{n=1}^{\infty}\left(1-x^{n} y^{n}\right)\left(1-x^{n} y^{n-1}\right)\left(1-x^{n-1} y^{n}\right) & \\
& =\sum_{n=-\infty}^{\infty}(-1)^{n} x^{n(n+1) / 2} y^{n(n-1) / 2} .
\end{aligned}
$$

Now let $\beta(n, m)$ denote the number of partitions of $(n, m)$ into (not necessarily distinct) parts

$$
(a, a),(b, b-1),(c-1, c) \quad(a, b, c=1,2,3, \cdots) .
$$

Then (6) implies

$$
\sum_{r=-\infty}^{\infty}(-1)^{r} x^{r(r+1) / 2} y^{r(r-1) / 2} \sum_{n, m=0}^{\infty} \beta(n, m) x^{n} y^{m}=1 .
$$

Consequently

$$
\sum_{r}(-1)^{r} \beta\left(n-\frac{1}{2} r(r+1), m-\frac{1}{2} r(r-1)\right)=0 \quad(n+m>0),
$$

where the summation is over all $r$ such that

$$
\frac{1}{2} r(r+1) \leqq n, \quad \frac{1}{2} r(r-1) \leqq m .
$$

If we put

$$
\prod_{n=1}^{\infty}\left(1-x^{n} y^{n-1}\right)^{-1}\left(1-x^{n-1} y^{n}\right)^{-1}=\sum_{n, m=0}^{\infty} \gamma(n, m) x^{n} y^{m},
$$

so that $\gamma(n, m)$ is the number of partitions of $(n, m)$ into (not necessarily distinct) parts

$$
(a, a-1), \quad(b-1, b) \quad(a, b=1,2,3, \cdots),
$$

it is evident that

$$
\beta(n, m)=\sum_{r=0}^{\min (n, m)} p(r) \gamma(n-r, m-r) .
$$

\section{REFERENCE}

1. G. H. Hardy and E. M. Wright, An introduction to the theory of numbers, Clarendon Press, Oxford, 1938.

DURe University 